\title{
Innovations in Environmental Monitoring Using Mobile Phone Technology - A Review
}

\author{
http://dx.doi.org/10.3991/ijim.v8i2.3645 \\ M.J. Aitkenhead, D. Donnelly, M.C. Coull and E. Hastings \\ The James Hutton Institute, Craigiebuckler, Aberdeen, Scotland, UK
}

\begin{abstract}
In recent years, the use of mobile phones and tablets for personal communication has increased dramatically, with over 1 billion smartphones out of a total of 5 billion mobile phones worldwide. The infrastructure and technology underlying these devices has improved to a level where it is now possible to integrate sensor technology directly and use them to acquire new data. Given the available resources and the number of technical challenges that have already been overcome, it would seem a natural progression to use mobile communication technology for field-based environmental monitoring. In this work, we review existing technology for acquiring, processing and reporting on environmental data in the field. The objective is to demonstrate whether or not it is possible to use off-the-shelf technology for environmental monitoring. We show several levels at which this challenge is being approached, and discuss examples of technology that have been produced.
\end{abstract}

Index Terms-Environmental monitoring, mobile technology, apps, sensors, mobile phones.

\section{INTRODUCTION}

The potential of mobile phone technology to provide rapid, cost-effective environmental monitoring has expanded greatly in recent years. An important aspect of this is the fact that the millions of mobile phones currently being used around the world are the product of a mature technology that is mass-produced cheaply and reliably. These systems are designed to be flexible and act as miniature computers, to enable the user to carry out a number of different activities and also to allow developers to produce software, in the form of applications (apps) that can be installed easily and rapidly. This ready-to-use technological base, ubiquitous and purposefully designed to be extensible, provides fertile ground for environmental monitoring on a massive scale. Ref. [1] provides a broad survey of the technology being used for mobile phone sensing systems. Here we provide a more in-depth examination in relation to environmental sensing, focussing especially on the technological sophistication and user/device interaction level.

Environmental monitoring and healthcare are two areas that overlap in relation to sensing systems. Healthcare, particularly in developing countries or remote regions, can potentially benefit greatly from small, cheap sensors that can be plugged into mobile phones. Ref. [2] described a mobile phone add-on containing a sensor on a chip capable of detecting specific disease biomarkers rapidly and without requiring great expertise on the part of the user. Ref. [3] demonstrated a system that uses a mobile phone camera to detect and measure light levels in a fluorescing sensor chip. This kind of technology presents great potential for disease detection, biosecurity, food safety and environmental monitoring. Ref. [4] also demonstrated the use of mobile phone technology to relate activity and location (using built-in geolocation functionality of mobile phones to determine position and travel speed) to air pollution information, and showed that this technology could be used to provide an unobtrusive method of environmental monitoring for health purposes.

Many examples of technology in this area already exist. Ref. [5] discusses the potential for using mobile phones for environmental sensing, although they focussed on sensors embedded into the mobile phone rather than augmentation with additional devices. Work at The James Hutton Institute on linking statistical models to mobile phone apps with image analysis techniques has led to rapid field monitoring for soil [6].

Here we identify and review a number of examples over a wide range of technological sophistication and level of user/device interaction, in order to provide a summary (with notable examples identified) of what is currently available. We have focussed on instrumentation and software that uses mobile phone/tablet technology, rather than on small-scale computers such as the Raspberry Pi or Arduino or on large-scale automated monitoring systems that use mobile phone/GPRS networks for remote data transmission, as the focus is on technology that can be implemented 'off the shelf'. This work is based upon an earlier report [7] developed for SEPA (Scottish Environmental Protection Agency) that focussed on aquatic monitoring, but we have broadened the scope to cover environmental monitoring in general.

\section{MethodS \& RESUlTS}

\section{A. Categorisation of technology}

There are many different solutions available within the field of environmental monitoring, with many aimed at human health, water quality and agriculture in particular. Grouping the different technological and computing developments into specific categories allows us to more easily compare these different solutions, and allows the reader to focus on the category that is of greatest interest to them. There are several possible frameworks of categorisation of mobile environmental monitoring solutions. We have chosen to group them in two ways: by technological sophistication (which is assumed to be closely related to cost) and by user/device interaction. The terms used in the listings below are our own, and are intended to reflect the category in each case while providing headings for a table in the Discussion. 


\section{1) Technological sophistication}

- Observation-only apps - purely app-based, with no additional technology required and little or no interpretation of the information obtained. Commonly used to record imagery of events or specific features within the landscape, sometimes with the option to provide additional data from the user's visual interpretation (e.g. water quality, presence of a particular species, see later examples).

- Clever apps - again, purely app-based but with more sophisticated interpretation of information obtained by the user. An example could be where image analysis is integrated with additional knowledge available from knowing the user's geographical location and both are used to drive models or to provide information directly to the user from digital maps.

- Plug \& play - off-the-shelf sensor devices that can be attached externally onto a mobile phone or tablet and used to augment the sensor technology of the device. These are ready to use and provide specific sensor readings.

- Clever kits - either for purchase in kit form, or recently developed and intended for testing and later development as a 'plug and play' device, this type of technology requires construction and a high degree of understanding from the user. Sensor devices of this kind are usually customisable and are designed to be used by researchers or professionals.

\section{2) User/device interaction}

- Crowd source - here, the user is not required to provide any interpretation of the results, but simply acts as a recorder of information (usually imagery, but sometimes audio recordings). The information is transmitted to a central location where it can be subjected to further interpretation by experts. This level of interaction can potentially take place at any of the levels of technological sophistication described above, although it tends to be seen more at the 'observation-only app' level.

- Personal use - the user is provided with readings, usually interpreted automatically to make them more useful, about their environment. There is no expertise required in handling the equipment or interpreting the information obtained, but some experience in using the equipment properly might be necessary.

- Decision support - information is taken from the user, either in the form of imagery for interpretation or numerical values (or both). This is integrated with information derived from knowledge of the user's position within the landscape, and is used to provide information that is of specific interest for land management etc. The outputs from this type of system tend to be less 'interpreted' than they would be for a member of the public.

- Mobile lab - the user of this technology is a specialist, often a scientist or someone looking to answer a specific question that requires sophisticated monitoring. Use of this technology requires an understanding of the system being monitored, training in the use of the technology and the ability to interpret sophisticated monitor readings. The use of the term 'tricorder' for this kind of multifunctional sensing device is in- creasingly common in the scientific and medical community in relation to smartphone use (e.g. [8]).

The following table shows how the above categorisation works, and lists the examples that we have been able to find that fall into each category, with links to a discussion of each example.

TABLE I.

CATEGORISATION OF SOLUTIONS, WITH EXAMPLES

\begin{tabular}{|c|c|c|c|c|}
\hline & $\begin{array}{c}\text { Observation-only } \\
\text { apps }\end{array}$ & Clever apps & $\begin{array}{l}\text { Plug \& } \\
\text { play }\end{array}$ & Clever kits \\
\hline $\begin{array}{l}\text { Crowd } \\
\text { source }\end{array}$ & $\begin{array}{l}\text { What's invasive } \\
\text { NatureWatch }\end{array}$ & $\begin{array}{l}\text { Water Qual- } \\
\text { ity Reporter }\end{array}$ & & \\
\hline Personal use & PEIR & & $\begin{array}{l}\text { Lapka } \\
\text { Ghana air } \\
\text { quality } \\
\text { Thermodo } \\
\text { Alcohoot }\end{array}$ & \\
\hline $\begin{array}{l}\text { Decision } \\
\text { support }\end{array}$ & $\begin{array}{l}\text { SoilWeb } \\
\text { SoilMapp } \\
\text { mySoil }\end{array}$ & $\begin{array}{l}\text { SIFSS } \\
\text { SOCiT }\end{array}$ & & $\begin{array}{l}\text { Platforma } \\
\text { SINC } \\
\text { MoboSens }\end{array}$ \\
\hline Mobile lab & & $\begin{array}{l}\text { EMAP } \\
\text { SEPA } \\
/ \text { Geofield }\end{array}$ & $\begin{array}{l}\text { Tesla } \\
\text { Micro } \\
\text { Proscope }\end{array}$ & $\begin{array}{l}\text { Sensordrone } \\
\text { WiMoto } \\
\text { smarTROLL }\end{array}$ \\
\hline
\end{tabular}

\section{B. Presentation of relevant examples}

We have presented below a list of examples that have been found of apps, mobile phone-related equipment and associated systems for environmental monitoring. The relevance of these examples varies, and where necessary we have identified the concepts or ideas that are relevant to environmental monitoring and that could be extracted for future systems. In each case, we have identified the design function, the potential of the system for environmental monitoring, the level of technological sophistication, the level and type of user/device interaction, any limitations on the use of the system in question and its suitability (i.e. the level to which it achieves the stated purpose).

\section{Name: What's Invasive}

Web address: http://whatsinvasive.com/

Designed function: Crowd-sourcing of invasive plant species.

Environmental monitoring potential: One of a number of community data collection apps, it is designed for use in collecting observations on the locations of invasive species of plants. It could equally be applied to animals, and is applicable in terrestrial or aquatic settings, and across a wide range of habitat types or ecosystems. Indirectly, this app and others similar to it can provide a template for capturing user observations of features or issues of interest e.g. pollution incidents, algal blooms, illegal dumping etc.

Technological sophistication: The app is relatively simple and acts more as a mechanism for capturing user observations than anything else. Photographs can be geotagged automatically, as can observations, allowing databases to be populated with user observations and images. However, there is no automated interpretation of the information sent, neither is there much in the way of moderation - the quality or relevance of an observation varies with the user. 
User/device interaction: This app can be used with the minimum of training or experience, and requires no sophisticated technical knowledge on the part of the user. However, they are expected to be able to identify the species or other feature of interest that they are describing.

Limitations on use: This is very much a 'what goes in is what comes out' app - all information of use is entered by the users, whose level of expertise in the subject area may vary. It is difficult therefore to produce statistical or numerical evaluations of the topic of interest.

Suitability assessment: Potentially useful for capturing the spatial location and distribution of qualitative observations. Not capable of capturing quantitative observations unless used in parallel with monitoring equipment. In order to make use of this app for monitoring environmental quality more generally, it would have to be developed to allow different observations to be entered.

\section{Name: PEIR}

Web address: http://www.cs.cornell.edu/ destrin/resour ces/conferences/2009-jun-Mun-Sheddy-PEIR.pdf

Designed function: Developed at UCLA and demonstrated in 2009, which calculated exposure to particulate matter in cities based on positional information and mode of transportation.

Environmental monitoring potential: Assessing user risk exposure to airborne pollutants is useful by itself, and the methodology could be transferred to other types of hazard e.g. water-borne pollutant exposure assessment.

Technological sophistication: Records the user's position and movement automatically, and integrates this information with existing datasets. The results are interpreted for the user and presented in an understandable form.

User/device interaction: Relatively simple user interaction is involved - the app presents the user with a set of results about air pollution exposure and also sends this information to servers for later data interpretation.

Limitations on use: Only designed for airborne pollution currently, but could be enhanced to be applicable to water and other forms of environmental pollution relatively easily.

Suitability assessment: Designed to integrate information about the user's position with existing spatial datasets and expert knowledge about transport systems and air pollution. As such, it requires existing datasets to be available, so wider use would only be feasible when assessing exposure to pollutants that have already been mapped or can be modelled.

\section{Name: Water Quality Reporter}

Web address: http://www.bristol.ac.uk/aquatest/inaction/aquatest-system/wqr/

Designed function: Transmits information from a water testing device to water supply companies.

Environmental monitoring potential: This app is designed specifically to allow water monitoring results to be sent to a central system for processing, and has been extensively tested and used in the field. The measurements uploaded by the user are perhaps not the full range that could be applied.

Technological sophistication: Relatively easy to use and technologically unsophisticated - this app is designed to be used by anyone in the field who has access to the relevant water quality monitoring equipment and a mobile phone. The data recording side is quite well designed and provides a framework for later remediation and interpretation.

User/device interaction: Straightforward data input by the user - no connection between the monitoring device and the mobile phone.

Limitations on use: The user has to upload the measurements, rather than having a direct connection between the monitoring device and the mobile phone or tablet. This means that there is a time/effort constraint on uploading multiple measurements.

Suitability assessment: This app is suitable for water quality monitoring, but is fairly restrictive in that it requires user input rather than connection to the monitoring equipment, and limits the types of observations that can be entered. By connecting to different types of sensors it could have application in more general pollution monitoring, contaminated land assessment, and risk exposure studies.

\section{Name: Platforma SINC}

Web address: http://www.alphagalileo.org/ViewItem. aspx? ItemId $=128267 \&$ CultureCode $=$ en

Designed function: Equipment can be used to detect mercury concentrations in water, based on colour change in sensitive paper. Not fully implemented as a monitoring tool, but its use with mobile phones has been demonstrated.

Environmental monitoring potential: Good potential, needs to be more fully developed to be an integrated package. Some kind of standardised detection mechanism (i.e. standardized chemosensitive paper in a mechanism that allows easy imaging) would improve the functionality.

Technological sophistication: Relatively low-tech, although the image analysis software requirements for detection and processing of the chemosensitive paper colour change are not trivial.

User/device interaction: Some activities required on the part of the user, in relation to sample preparation and acquisition of appropriate imagery.

Limitations on use: The limitations on this kind of application relate more to the chemosensitive paper (or whatever sensing mechanism is used that changes colour). If there is a chemical of interest and a reliable colourchange chemistry that can be implemented in the field, then there are no limitations on application.

Suitability assessment: Currently most suitable for water pollution monitoring, but if appropriate chemicalcolour change mechanisms can be implemented that are cheap, rapid and accurate, a wider range of pollution and contaminated land assessment would be possible.

\section{Name: Ghana Air Quality}

Web address: http://www.globalproblems-globalso lutions-files.org/unf website/PDF/vodafone/tech social change/Environmental_Conservation_case3.pdf

Designed function: Carbon monoxide sensor linked to mobile phone technology, used as a pilot study.

Environmental monitoring potential: The sensor that links to the mobile phones was designed specifically to record atmospheric carbon monoxide levels. However, similar sensors could be developed with effectively identical electronic interfaces to the mobile phone technology. 
PAPER

Innovations in Environmental Monitoring Using Mobile Phone Technology - A Review

Technological sophistication: The sensing device is the most sophisticated part of this system, with the recording app being relatively simple and low in 'options'.

User/device interaction: No user/device interaction, this is a portable recording device that the user (a taxi driver) transports in their vehicle and which records information about air quality over time. The recorded information is uploaded to servers for later processing.

Limitations on use: The limitations relate to the physical monitoring mechanisms - a water quality sensor that produces a translatable electronic signal could be linked to a mobile phone just as easily as a sensor that detects air pollution levels. However, such a sensor does need to exist in the first place.

Suitability assessment: Potentially suitable for environmental monitoring, if the monitoring equipment is available. An app for recording readings could be produced rapidly and cheaply.

\section{Name: Sensordrone}

Web address: http://www.kickstarter.com/projects/ 453951341/sensordrone-the-6th-sense-of-yoursmartphoneand-be

Designed function: Multisensory device for mobile phones, for use in experimental environmental monitoring.

Environmental monitoring potential: This system has a lot of potential as it allows sensors that measure relevant parameters to be linked through Bluetooth technology to mobile phones, and provides a 'whole package' system for acquiring, storing and visualizing logged data.

Technological sophistication: This is a relatively sophisticated system, as it includes sensors, connectivity to mobile phones for download and storage of measurements, and apps for visualizing and controlling this information.

User/device interaction: While a lot of the information acquisition and storage is automated, there is still a degree of user/device interaction required in setting up the system for use and in ensuring that the observations are recorded properly.

Limitations on use: Currently, there are only a small number of sensors available, although this situation is changing rapidly. The user is therefore limited to using these specific sensors and cannot plug in a different sensor that may be of more relevance to their interests.

Suitability assessment: Highly suitable within a range of parameters. The system provides a complete package for rapid observation of the environment, although it does require quite a high level of understanding and implementation on the part of the user.

\section{Name: EMAP}

Web address: http://www.aecom.com/What+We+Do/ Environment/Practice+Areas/Impact+Assesment + and + Permitting/Environmental+Mobile+Application+for + Pro jects

Designed function: Geographical Information System (GIS) package for mobile tablets, designed to allow information to be captured, edited and processed in the field. Designed as an all-in-one package that facilitates environmental monitoring at all stages from field data capture to reporting, all from a single mobile platform.
Environmental monitoring potential: Extremely useful as a mechanism for recording, processing and reporting. Not designed to allow monitoring devices to link directly to the tablet, so data entry is carried out manually.

Technological sophistication: A very sophisticated package, requiring a high level of user understanding, but with a lot of tools to allow field monitoring to be carried out more rapidly.

User/device interaction: The user interacts with the software package in a similar manner to that in which a GIS package would be used on a desktop. Additional functionality for reporting and filling in of technical sheets is also built in.

Limitations on use: The technology is proprietary to AECOM, a large American technical and management support company. Licensing may or may not be possible.

Suitability assessment: Extremely suitable for a wide range of environmental monitoring applications.

\section{Name: SEPA/Geofield diffuse pollution assessment}

Web address: http://www.sepa.org.uk/about us/news/ 2013/sepa_wins_connect_ict innovate.aspx?lang=en-gb

Designed function: Use of off-the-shelf tablets and sensor recording software in water monitoring. The application of this system is broad and has not been fully explored, as it allows users in the field to record a large number of different readings and to integrate these with existing spatial datasets.

Environmental monitoring potential: This system appears to have been designed to facilitate the recording and later interpretation of a wide range of environmental monitoring. Its potential lies therefore in assisting with the input, organisation and archiving of readings rather than the direct acquisition of the readings themselves.

Technological sophistication: High levels of technological sophistication are applied in the software that is used for the recording and organisation of sensor readings in the field. However, there is no mechanism for the user to integrate monitoring devices directly into the system and to record the readings without the human interface.

User/device interaction: The user must enter measurements into the system, which does not provide any feedback or processing but which does allow visualization of the data entered.

Limitations on use: The main limitation here is the user themselves - if a system could be developed that could accept data directly from a number of recording devices, then the user could act in a much more effective 'oversight' capacity rather than having to be directly involved in the measurements and their recording.

Suitability assessment: Highly suitable for monitoring of water and environmental pollution, and for a number of other monitoring requirements. The suitability lies more with the system's facilitation of data entry and recording, and with allowing later assessment, than with the direct recording of sensor readings or the integration of readings with existing data to provide 'added value'.

\section{Name: NatureWatch}

Web address: http://www.eea.europa.eu/mobile

Designed function: Allows citizen scientists to identify and report invasive species

Environmental monitoring potential: Similar to the "What's Invasive" app 
Technological sophistication: Similar to "What's Invasive", but the user can also submit video and audio clips that could be of value in recording presence of invasive animals, birds, insects etc.

User/device interaction: This app can be used with the minimum of training or experience, and requires no sophisticated technical knowledge on the part of the user. Guidance is given on recognizing invasive species.

Limitations on use: This is very much a 'what goes in is what comes out' app - all information of use is entered by the users, whose level of expertise in the subject area may vary. It is difficult therefore to produce statistical or numerical evaluations of the topic of interest.

Suitability assessment: Potentially useful for capturing the spatial location and distribution of qualitative observations. Not capable of capturing quantitative observations unless used in parallel with monitoring equipment. Otherwise, similar limitations to "What's Invasive".

\section{Name: SIFSS}

Web address: http://sifss.hutton.ac.uk/

Designed function: Allows a user to find information about soil at their location (using the unit's GPS or interactive map) within Scotland and get a range of chemical and physical parameters such as $\mathrm{pH}$, carbon content, texture, nitrogen, phosphorus, and base cations.

Environmental monitoring potential: A wide range of potential application e.g. assessment of nutrients and eutrophication - soil nitrogen \& phosphorus are available in the app. Future developments are likely to include reporting on whether the user is in a Nitrate Vulnerable Zone (NVZ) and the HOST class (Hydrology of Soil Type) of the soil.

Technological sophistication: The most comprehensive of existing UK soils apps (albeit with information for Scotland only). Detailed information on up to 15 soil characteristics from the Soil Survey of Scotland are provided for each soil horizon, for both cultivated and seminatural land covers (where they exist). The app accesses James Hutton databases, so always points to the most up to date data.

User/device interaction: This app can be used with the minimum of training or experience, and requires no sophisticated technical knowledge on the part of the user. Where several soil series are present at a location, the user is given a description of the appearance of the soil as a guide to selecting the correct series. No user information is incorporated in the app.

Limitations on use: Currently uses only the Scottish soil mapping information at 1:250,000, but future versions will incorporate higher resolution mapping where it is available. Could be adapted easily for other countries where relevant information is available.

Suitability assessment: Suitable for any field-based assessment that requires the type of information accessible through the app.

\section{Name: SOCiT}

Web address: http://www.hutton.ac.uk/research/groups/ information-and-computational-sciences/esmart

Designed function: SOCiT provides the user with a near instantaneous field assessment of soil organic matter content using image analysis and modelling.
Environmental monitoring potential: Apart from determining soil carbon, potentially very useful conceptually the integration of sensor information and environmental data sets in near-realtime.

Technological sophistication: From the perspective of the user this is quite a simple app to use, requiring only the device's camera (and a spade). However, there is a lot of server-side processing that integrates automated image analysis and neural network-based environmental data analysis.

User/device interaction: The user has to dig a hole, drop an image normalization card in it, take a photo and click a button for the soil organic matter to be calculated.

Limitations on use: Currently Scotland only (due to access to environmental data sets), potentially extending to rest of UK in the future.

Suitability assessment: At present this is a soil information tool but could provide a template for other apps integrating image and environmental data analysis.

\section{Name: SoilWeb}

Web address: http://casoilresource.lawr.ucdavis.edu/ soilweb/

Designed function: Provide access to soil mapping information from the United States. This includes drainage class, water table depth and available water storage (in soil).

Environmental monitoring potential: Has potential in the US as detailed soil mapping is used in addition to the soil hydrology information.

Technological sophistication: This appears to be similar to SIFSS, but with more restricted user control of location selection - this app permits only input by the device GPS.

User/device interaction: Minimal "Get My Location" information required, which is automatically achieved.

Limitations on use: US only - blocked for Android distribution outside US and while available for Apple devices the only input is via the device's GPS.

Suitability assessment: Limited.

\section{Name: SoilMapp}

Web address: http://www.csiro.au/soilmapp

Designed function: Provides access to soil mapping information from Australia. This includes maps, photographs, satellite images, tables and graphs of data about nearby soils. Also includes soil's physical and chemical characteristics, including acidity $(\mathrm{pH})$, soil carbon, available water storage, salinity and erodibility.

Environmental monitoring potential: Potentially useful due to the large number of attributes linked to soil hydrology, but only in Australia.

Technological sophistication: This is an interactive map, but gives access to very large amounts of data in a convenient manner.

User/device interaction: The user interacts with the soil map displayed on the device to obtain information. Information on site conditions can also be uploaded to CSIRO by users for subsequent use in map validation.

Limitations on use: Available for iPad only, data for Australia only.

Suitability assessment: Limited, but a good example of what can be done, and potentially useful in guiding future developments of existing apps. 


\section{Name: mySoil}

Web address: http://www.bgs.ac.uk/mysoil/home.html

Designed function: To provide access to soil mapping information from the majority of Europe. This app provides extensive coverage of soil information, but with a limited number of attributes, and low precision (e.g. Organic Matter: "Medium" and pH: "Slightly Acidic")

Environmental monitoring potential: With current information available in the app quite low, however, it is likely that more data sets will be added in the future.

Technological sophistication: Fairly limited, a clickable interactive map.

User/device interaction: Uses a clickable interactive map with web mapping services (WMS). Users can also upload their observations which are subsequently displayed as pushpins.

Limitations on use: Europe only. While the extent of the cover within Europe is comprehensive, the depth of information being provided is quite low.

Suitability assessment: The app contains information from the UK's Countryside Survey and the Land Cover Map (2007). It is possible that other aspects of the data from these surveys, if incorporated in future versions (or new apps), could make this app more useful in monitoring or assessment.

\section{Name: Lapka}

Web address: https://mylapka.com/

Designed function: Lapka Personal Environment Monitor is a collection of sensors (which fit together in a wood and moulded plastic block) which interface with an iPhone via the associated app. The sensors are individual devices which plug into the audio jack of the phone and measure electrical conductivity (which they claim is highly correlated to nitrate concentration so is billed as a 'food quality' monitor), humidity and temperature, radiation, and electromagnetic fields.

Environmental monitoring potential: Depending on how sensitive the sensors are, having the capability of measuring electrical conductivity and temperature in one device which interfaces directly to the phone (thus allowing records to be associated with geotagged photographs) is appealing on the face of it. How the device would match up against purpose built sensors is unknown without testing. The package is sold for \$220 (USD) which the makers claim compares favourably with the cost of a single humidity sensor at around $\$ 200$, but puts it outside the range of most citizen scientists unless provided by a third party.

Technological sophistication: The various sensors are all available in other forms (e.g. Thermodo for temperature), but the package appears to be much more about style than function.

User/device interaction: Simply plug each sensor into the phone and the app records the appropriate measurement.

Limitations on use: Cost, sensors not especially sensitive or measuring different things to other devices.

Suitability assessment: Moderate/Low. Limited range of useable information, although having multiple sensors in one device is a plus.

\section{Name: Tesla Microscope}

Web address: http://tesla.dma.ucla.edu/TEST6/?q=re search/fluorescent-microscope-cell-phone-attachment

Designed function: This device is a 'matchbox-sized attachment that converts a mobile phone's camera into a fluorescent microscope. The device utilises an inexpensive lens and battery-powered, light-emitting diodes to create a field of view some two orders of magnitude larger than previous cell-phone fluorescent microscopy technology. It is more than five times smaller than previous cell phone microscopes.

Environmental monitoring potential: The device is designed to be able to quickly and accurately analyze large sample sizes. The proposed use is in testing medical samples in remote areas, but the technology would easily lend itself to analysis of water and other fluids for pathogens. The inventors state that while it does not have the resolution of conventional microscopes, it is high enough to 'screen for labeled pathogens in drinking water or food and to image various body fluid samples to search for disease markers'.

Technological sophistication: Extremely high, although it appears to have been designed for size and robustness in the field. No indication if it being mass-produced or if it is still at the experimental design stage.

User/device interaction: Relies on a high degree of user input and interpretation.

Limitations on use: Some other equipment required (e.g. a colour filter), although this device is designed to be able to work with cheaper alternatives to the usual filters.

Suitability assessment: Potentially very suitable. Individual users would need training before it could be rolled out.

\section{Name: Proscope}

Web address: http://www.bodelin.com/proscope/pro scope-micro-mobile

Designed function: A professional quality microscope which attaches to an iOS device. This relatively inexpensive device (c\$150 (USD)) fits onto an iOS device and provides $20 \mathrm{X}$ to $80 \mathrm{X}$ magnification, with high quality optics. Includes stand, integrated LED lighting, and allows photographs of the subjects to be taking using the devices native camera.

Environmental monitoring potential: Potentially extremely useful for in the field monitoring of invertebrates and other biological specimens. Adding of geotagged photographs has potential for rapid biological field assessments.

Technological sophistication: High. Produced by a company with a track record for portable and/or internet enabled microscopes. Robust design specifically designed for field operation.

User/device interaction: Some familiarization with the equipment required (as well as the specific skills required to carry out the task in question).

Limitations on use: Interpretation skills of user

Suitability assessment: Wide range of potential applications

Name: Thermodo

Web address: http://thermodo.com/

Designed function: An external temperature sensor for a smartphone, tablet 
Environmental monitoring potential: The device (which interfaces with an app from the same developer) is a very small temperature sensor which plugs into the audio jack of the phone. While it is designed to measure air temperature, there seems to be no reason why you could not plug an extension into the audio jack, and dangle the sensor itself in water or any semi-liquid medium such as slurry, food products etc. While it offers no real benefit over using a standard thermometer, as the app will (presumably) geotag the locations where the measurements are made, it would cut down on data processing time in the office plotting temperature readings spatially/temporally.

Technological sophistication: Medium/Low. The sensor itself is the most sophisticated part, although affordable (c\$30 USD))

User/device interaction: Simply plug in the device, start the app and record.

Limitations on use: No better than a standard thermometer. Even if solution for working in liquids proposed above is workable, phones are not very robust so care would have to be taken in the field not to damage it.

Suitability assessment: Limited, but also shows the capability for developing small sensors which plug into smart device and can interface with a simple app.

\section{Name: Alcohoot}

Web address: http://www.getalcohoot.com/

Designed function: Measurement of blood alcohol concentration (BAC)

Environmental monitoring potential: This is a small sensor device which attaches to a smartphone (compatible with iPhone, iPad and Android devices currently) via a $3.5 \mathrm{~mm}$ jack. Its intended purpose is to measure the user's blood alcohol content in the same way as a conventional breathalyzer. It uses a fuel cell sensor (similar to the type used in police breathalyzers) as opposed to a semiconductor sensor, which allows it to be specific to alcohol (and unaffected by other solvents or chemicals such as hairspray, deodorant etc), as well as a patented air sensor to detect airflow into the device. If the sensor could be replaced with different kinds, then it might be possible to use as a relatively sophisticated air quality detector for measuring point source gas emissions which might help in tracing of chemical leaks, spillages etc.

Technological sophistication: The internal sensors are quite sophisticated, although the device itself retails for $\$ 75$ (USD) so is still affordable. For the BAC measurements, the sensor needs to be periodically recalibrated which either would require return to base (RTB) or provision of some method for the user to do it in the field.

User/device interaction: The user simply connects the device to the phone and blows into the mouthpiece.

Limitations on use: Need for periodic calibration.

Suitability assessment: Limited to blood alcohol content currently, but shows the capability of designing and implanting add-on sensors for specific applications. The challenge would be to create small, accurate and reliable enough versions of other gas sensors for this to be a useable technology for pollution monitoring.

\section{Name: WiMoto}

Web address: http://www.wimoto.com/

Designed function: A variety of small wireless sensors which can measure ambient temperature, humidity, light, soil moisture, soil temperature, object temperature, human presence and movement.

Environmental monitoring potential: Unlike a number of the other sensor add-ons in this review, these can send their data wirelessly via Bluetooth. Assuming they are robust enough for deployment in the field, they could replace the need for manual download of temperature loggers and/or allow placement of the sensors in locations where accessing loggers frequently would be hazardous or time-consuming. The example use suggested for the temperature sensor on their crowd funding page is to monitor a swimming pool.

Technological sophistication: The sensors themselves appear no more complex than others mentioned here but the addition of battery powered Bluetooth is different.

User/device interaction: All data collected via a smartphone or tablet, which can then be uploaded to a cloud server. Potentially allows data to be collected more quickly and easily than traditional in-situ loggers.

Limitations on use: Battery powered, the makers claim that batteries last for about a year. Uses Bluetooth SMART technology so receiving device needs to be capable of acquiring signal.

Suitability assessment: Potentially very high, especially for light and temperature in water. Still at the crowd funding stage, but could be used to develop other sensors using similar infrastructure e.g. dissolved oxygen, specific chemicals.

\section{Name: MoboSens}

Web address: http://nanobionics.mntl.illinois.edu/ mobosens/\#

Designed function: A smartphone based sensor add-on which can accurately measure nitrate concentrations in water due to be released by end of 2013. Development of arsenic sensor underway, with plans in place to expand to sensors covering other pollutants (specifically heavy metals).

Environmental monitoring potential: Specifically designed to tackle a major diffuse pollution issue.

Technological sophistication: Sensor prototype has been developed at Illinois University. Further development and testing at the crowd funding stage.

User/device interaction: Not known - this is still at the testing stage with pilot citizen science group/crowd funders, but assumed to be simple given purpose.

Limitations on use: Not known. Some training presumed, and no information given about where data would be uploaded and analyzed beyond the pilot area.

Suitability assessment: Very high. If the technology can be expanded to detect a wider range of determinands, then it would be the ideal device for large scale, crowd sourced pollution data collection, particularly of water bodies.

\section{Name: smarTROLL}

Web address: http://www.in-situ.com/products/waterquality/handheld-systems/smartroll-multiparameterhandheld

Designed function: This app is designed to interface with the smarTROLL water quality probe produced by InSitu Inc. The probe can measure dissolved oxygen, $\mathrm{pH}$, ORP, conductivity (actual or specific), salinity, total dissolved solids, resistivity, and density, as well as physical parameters such as air and water temperature, barometric 
pressure, water level, and water pressure. The phone app replaces the need to have a handheld meter or logger attached to the probe, and allows data to be sent instantly to the office or uploaded to the cloud subject to having a phone signal.

Environmental monitoring potential: Specifically designed for this purpose.

Technological sophistication: Low for the app itself as it only records readings made by the probe.

User/device interaction: Very simple, no real user knowledge required as the probe (assuming correct calibration) can be put in water and readings taken without any assumed skill or technical expertise. Eliminates need to download separate logger and potential transcription errors from manually adding data.

Limitations on use: Specific to the sensor, which is expensive. No capability for expansion as it would require the probe itself to have extra sensors added.

Suitability assessment: Very high for the specific attributes measured.

\section{DISCUSSION}

Most of the examples given above relate directly to the monitoring of one aspect of the environment, but in many cases the technology and software in question could be easily altered for use in a range of environmental monitoring tasks. In each case, a level of investment and redesign would be required. However, we emphasize that this investment is quite small in nearly every case - there are a lot of solutions available that would be very appropriate with some tweaking, and in each case most, if not all, of the technological barriers have been overcome through earlier development work.

Gaps in the above table show a trend that is not difficult to explain - the more sophisticated and/or expensive the technological application, the more likely it is to be used for scientific or industrial research. Complex equipment requiring experienced users is not used in citizen science, while purely app-based recording of simple observations does not occur with sophisticated monitoring of complex environmental conditions.

A notable example is the application of SEPA's existing Geofield framework for recording, organising and later processing of environmental monitoring readings using tablets and geospatial software developed by SigmaSeven (http://www.sigmaseven.com/geofield). What SEPA appear to have in this case is a system that solves half of the problem (we are not being disparaging here this is half of a very big problem) and that facilitates a wide range of environmental monitoring. In order to take this system which is already in place and appears to work well a step further, it is necessary to remove the human element, or to at least reduce the amount of human data input required. Automated sensor acquisition using Bluetooth technology or $\mathrm{Wi}-\mathrm{Fi}$ download would require a fair degree of further development in the data recording interface, but would enable measurements to be made more rapidly and would remove the risk of human error. GPRS (General Packet Radio Service) is another potential option here, although it may not always available in the field.

The systems that we have discovered in our search that may provide this 'missing element' are the Sensordrone, WiMoto and smarTROLL. While nowhere near as sophis- ticated in terms of presentation and immediate user ease of use as the Lapka, these systems are highly flexible and generalizable, and furthermore are to a certain degree 'programmable', i.e. the sensor readings can be controlled by the user in a manner that is not available through other systems. The developers of Sensordrone in particular have produced a system that is at this end of the development scale, with a wide range of potential applications that integrates well with mobile phone technology and produces useful information. However, the requirement for user expertise in achieving the potential of Sensordrone is a likely stumbling block. Comparison of the Sensordrone webpage with that produced by Lapka is the first sign that here we have something designed with scientists and 'early adopters' in mind, rather than members of the general public interested in knowing about potential contaminants in food that they have purchased. The other two systems, WiMoto and smarTROLL, lie somewhere between these two extremes.

Additional systems of note include the following:

- PEIR - a useful example of integrating automatically recorded sensor data with position and existing spatial datasets of relevance. The image below is extracted from the referenced link, as is the case with images relating to the other examples given.

- Water Quality Reporter - an example of a relatively simple, widely applicable app for uploading of observations. This concept could be readily and rapidly applied to almost any kind of water-related monitoring.

- Platforma SINC - cleverly applies colour interpretation from the mobile phone camera of sensor strips. A relatively low-tech but potentially rapid and simple tool.

- EMAP - this tablet-based GIS package is similar in concept to the system currently being used by SEPA and developed by Geofield. A very useful system for facilitating monitoring in the field, although the next obvious step (removing the human input requirements and connecting directly to sensors) needs to be made.

- Tesla microscope - a microscope attachment for mobile phones that could be used for monitoring waterborne bacteria, requires user expertise in identification but potentially extremely useful.

- Proscope - similar to the example above, with a few additional features including geotagging of images and easier recording of video.

- MoboSens - this system is not available yet and details are on the scant side, but appear to promise a wide range of specific sensors that can connect to mobile phones.

In many cases, the examples of mobile phone technology for environmental monitoring that we have reviewed in this work are still in the testing stage and require the user to 'assist'. This can involve either providing a mechanism for getting the sensor into the medium that is being tested (e.g. by dipping a colorimetric sensing material into water, [9]), or by providing interpretation of the raw recorded data (e.g. contextualising noise levels, [10]). In addition, for large-scale environmental monitoring using the millions of potential recording devices to actually take place, 
user (or in this case, device owner) participation must be achieved. Ref. [11] discusses potential incentive methods for accomplishing this. A step-change is required both in terms of automating data capture (either through augmenting the current sensor suite of a mobile phone, or finding ways to more cleverly turn data captured through existing sensors into useful information), and in engaging participants, such that they are willing and able to use their personal devices in this way. This last point will require consideration of personal privacy, data source anonymity and potential misuse or unwanted (by the people involved) use of the data that is captured. A final point, as discussed by [12], is that even if useful real-time environmental monitoring data is available, either from dedicated field researchers or crowd-sourced from the general population, we still lack ways of effectively communicating the appropriate information to people that might benefit from it. Ref. [13] provides some pointers towards how a model of 'participatory public health' might emerge.

Another consideration that is important for most of the systems discussed here is that of data roaming limitations. Mobile phone signals are still weak in many parts of the world, particularly in areas of low human population density. An important aspect of systems designed for environmental monitoring is that they should be able to record while off-line, and use periods of available signal strength to upload observations when possible. The same is true of systems based on SMS messaging, which can be used to record observations but which still require a signal for transmission. Intelligent design of controlling apps that make use of signal strength opportunities, rather than being forced to attempt transmission of observations as soon as they are made, will make field-based observations more successful.

\section{CONCLUSIONS}

There are a number of different systems available on the market that are at least partially relevant for environmental monitoring, or that can inform developments in that direction. We have identified a number with particular promise, as well as categorizing the examples that we could find within a conceptual framework that enables comparison. Field-based environmental monitoring using off-the-shelf components is an area that is developing extremely rapidly, and trends in what is available would seem to indicate that more functionality and integration between sensors and mobile phone technology is highly likely in the near future. One aim of this paper was to demonstrate whether or not it is possible to use off-theshelf technology for environmental monitoring. We feel that given careful selection of the technology within a restricted range of observation types, that it is indeed possible to do this, and that ease of use and breadth of application are improving rapidly.

\section{ACKNOWLEDGMENTS}

The authors would like to thank Dr Peter Cock and Dr Paul Shaw of the James Hutton Institute for their advice in the preparation of this paper.

\section{REFERENCES}

[1] W.Z. Khan, Y. Xiang, M.Y. Aalsalem, Q. Arshad, "Mobile phone sensing systems: a survey", IEEE Communications Surveys and Tutorials 15(1), 402-427, 2013. http://dx.doi.org/10.1109/ SURV.2012.031412.00077

[2] P.B. Lillehoj, M.-C. Huang, N. Truong, C.-M. Ho, "Rapid electrochemical detection on a mobile phone", Lab on a Chip 13(15), 2950-2955, 2013. http://dx.doi.org/10.1039/c31c50306b

[3] S. O'Driscoll, B.D. MacCraith, C.S. Burke, "A novel camera phone-based platform for quantitative fluorescence sensing", Analytical Methods 5(8), 1904-1908, 2013. http://dx.doi.org/10.1039/ c3ay40116b

[4] A. de Nazelle, E. Seto, D. Donaire-Gonzalez, M. Mendez, J. Matamala, M.J. Nieuwenhuijsen, M. Jerrett, "Improving estimates of air pollution exposure through ubiquitous sensing technologies", Environmental Pollution 176, 92-99, 2013. http://dx.doi.org/10.1016/j.envpol.2012.12.032

[5] N.D. Lane, E. Miluzzo, H. Lu, D. Peebles, T. Choudhury, A.T. Campbell, "A survey of mobile phone sensing", IEEE Communications Magazine 48(9), 140-150, 2010. http://dx.doi.org/10.1109/ MCOM.2010.5560598

[6] M.J. Aitkenhead, "The E-SMART project", (http://www.hutton.ac.uk/research/groups/information-andcomputational-sciences/esmart), accessed $13^{\text {th }}$ March 2014.

[7] M.J. Aitkenhead, D. Donnelly, M.C. Coull, "Innovations in aquatic monitoring", CREW project number CD2013_04, Available online at: crew.ac.uk/publications.

[8] S. Novella, "Will your smartphone become a tricorder?", Sciencebased medicine (http://www.sciencebasedmedicine.org/will-yoursmartphone-become-a-tricorder/), accessed $5^{\text {th }}$ October 2013.

[9] H. El Kaoutit, P. Estevez, F.C. Garcia, F. Serna, J.M. Garcia, "Sub-ppm quantification of $\mathrm{Hg}(\mathrm{II})$ in aqueous media using both the naked eye and digital information from pictures of a colorimetric sensory polymer membrane taken with the digital camera of a conventional mobile phone", Analytical Methods 5(1), 54-58, 2013. http://dx.doi.org/10.1039/c2ay26307f

[10] E. Kanjo, "NoiseSPY: A Real-Time Mobile Phone Platform for Urban Noise Monitoring and Mapping", Mobile Networks \& Applications 15(4), 562-574, 2010. http://dx.doi.org/10.1007/s11036009-0217-y

[11] X. Sheng, X. Xiao, J. Tang, G. Xue, "Sensing as a Service: A Cloud Computing System for Mobile Phone Sensing", Proceedings $11^{\text {th }}$ IEEE Sensors Conference, 2012, Taipei, Taiwan, 22392242, 2012.

[12] F.J. Kelly, G.W. Fuller, H.A. Walton, J.C. Fussell, "Monitoring air pollution: Use of early warning systems for public health", Respirology 17(1), 7-19, 2012. http://dx.doi.org/10.1111/j.14401843.2011.02065.x

[13] D. Van Sickle, "The Next Generation of Public Health Approaches to Asthma in Asia and the Middle East", Asia-Pacific Journal of Public Health 22, 229-235, 2010. http://dx.doi.org/10.1177/ 1010539510373129

\section{AUTHORS}

M.J. Aitkenhead is with the James Hutton Institute, Aberdeen, Scotland, UK AB15 8QH (e-mail: matt.aitkenhead@hutton.ac.uk).

D. Donnelly is with the James Hutton Institute, Aberdeen, Scotland, UK AB15 8QH (e-mail: david.donnelly@hutton.ac.uk).

M.C. Coull is with the James Hutton Institute, Aberdeen, Scotland, UK AB15 8QH (e-mail: malcolm.coull@hutton.ac.uk).

E. Hastings is with the James Hutton Institute, Aberdeen, Scotland, UK AB15 8QH (e-mail: emily.hastings@hutton.ac.uk).

Submitted 13 March 2014. Published as re-submitted by the authors 05 April 2014. 